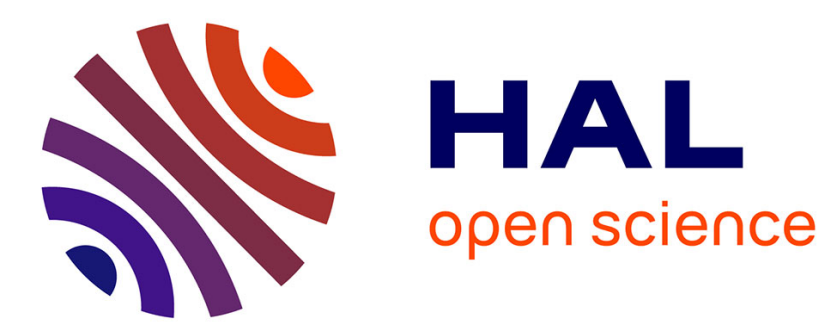

\title{
Sidewall and thermal boundary condition effects on the evolution of longitudinal rolls in Rayleigh-Bénard-Poiseuille convection
}

S. Mergui, Xavier Nicolas, S. Hirata

\section{> To cite this version:}

S. Mergui, Xavier Nicolas, S. Hirata. Sidewall and thermal boundary condition effects on the evolution of longitudinal rolls in Rayleigh-Bénard-Poiseuille convection. Physics of Fluids, 2011, 23 (8), pp.084101. 10.1063/1.3605698 . hal-00694578

\section{HAL Id: hal-00694578 \\ https://hal.science/hal-00694578}

Submitted on 4 May 2012

HAL is a multi-disciplinary open access archive for the deposit and dissemination of scientific research documents, whether they are published or not. The documents may come from teaching and research institutions in France or abroad, or from public or private research centers.
L'archive ouverte pluridisciplinaire HAL, est destinée au dépôt et à la diffusion de documents scientifiques de niveau recherche, publiés ou non, émanant des établissements d'enseignement et de recherche français ou étrangers, des laboratoires publics ou privés. 


\section{Sidewall and thermal boundary condition effects on the evolution of longitudinal rolls in Rayleigh-Bénard-Poiseuille convection}

S. Mergui, ${ }^{1}$ X. Nicolas, ${ }^{2}$ and S. Hirata ${ }^{2}$

1) UPMC Univ Paris 06, Univ Paris-Sud, UMR CNRS 7608. Lab FAST, Bat 502, Campus Univ, Orsay, F-91405, France.

2) Université Paris-Est, Modélisation et Simulation Multi Echelle, MSME UMR CNRS 8208, 5 bd Descartes, 77454 Marne-la-Vallée Cedex 2, France.

(Dated: 4 August 2011)

Experimental and numerical studies of steady longitudinal convection rolls that develop in a Poiseuille air flow in a rectangular channel heated from below and cooled from the top are conducted in the range $3500 \leq R a \leq 6000$ and $20 \leq R e \leq 200$. The effect of the lateral vertical walls on the onset and development of the convection cells is investigated by changing the transverse aspect ratio of the channel from 4.7 to 18.4. The influence of the entrance temperature and of adiabatic or conductive thermal boundary conditions at the side and top walls of the channel is also investigated. The scenario of the roll formation is described in details. It results in a symmetric pattern in the form of steady longitudinal rolls with an even number of rolls that depends not only on the aspect ratio but possibly on the inlet temperature of the flow. It is shown that the fully developed pattern is determined by the two rolls nearby each vertical side wall that are triggered just at the entrance of the channel due to the presence of velocity boundary layers adjacent to the walls. It is also shown that the heat conduction in the top horizontal wall of the experimental channel must be taken into account in the numerical simulations so that the experimental wavenumber can be properly depicted. 


\section{INTRODUCTION}

The thermoconvective motion in a horizontal fluid layer confined in a rectangular channel heated from below and cooled from above with a superposed laminar forced flow is a mixed convection flow designated as Rayleigh-Bénard-Poiseuille (RBP) convection. The onset and development of RBP flows have been extensively studied in the past few decades because this flow type has both fundamental and practical interests. A complete review of published works on RBP flows before 2000 was performed by Nicolas ${ }^{1}$ and a recent review on the RBP convection within the framework of the APCVD rectangular reactors (Atmospheric Pressure Chemical Vapor Deposition) is presented in ${ }^{2}$.

In experiments, parallel convection rolls oriented in the direction of the imposed pressure gradient are observed for Rayleigh numbers above a critical value and for sufficiently high Reynolds numbers (typically $R e>O(10)$ in air). These patterns are referred to as longitudinal rolls in contrast to transverse rolls with axis perpendicular to the mean flow detected for low Reynolds numbers. A first linear stability analysis conducted by ${ }^{3}$ for an infinite layer showed that longitudinal rolls are the preferred mode arising from the destabilization of the basic steady state (the purely conductive Poiseuille flow) for a critical Rayleigh number, $R a_{c}=1708$, independent of the Reynolds and Prandtl numbers. However, this result was in contradiction with all the experiments performed at low Reynolds number in channels of moderate transverse extent in which transverse rolls were detected $\left(^{4-6}\right)$. Linear stability analysis performed in a channel of finite transverse extent $b y^{7}$ and more recently extended by $^{8}$ have partially removed this contradiction by pointing out the stabilizing effect of the lateral boundaries and showing that transverse rolls are the preferred mode for low Reynolds numbers.

To go further in the characterization of the primary destabilization, theoretical and numerical investigations of the spatial development of the disturbances have been performed to detect transitions from absolute to convective instability for transverse or longitudinal patterns in an infinite layer $\left({ }^{9-11}\right) .{ }^{11}$ showed that a transition from convective to absolute instability occurs for the transverse rolls while the longitudinal rolls always result from a convective instability and that this mode is the most amplified one in channels of infinite lateral extension. Those results could explain why longitudinal rolls are the preferred patterns observed in real flows in the region where the transverse rolls are not absolutely unstable. 
But the origin of the perturbations required for triggering such a convective instability in uncontrolled experiments is not yet elucidated.

In real flow observations (that is in channels of finite lateral extension) a steady initiation mechanism is commonly reported $\left({ }^{12-16}\right)$. The longitudinal rolls are triggered in the vicinity of the side walls in the entrance zone of the heated flow and then develop step by step to the center as they move downstream in the channel. The resulting pattern at the beginning of the channel is illustrated in figure 1. This behavior could indicate that the side walls in the experiments plays the role of the perturbation allowing for the development of the convective instability.

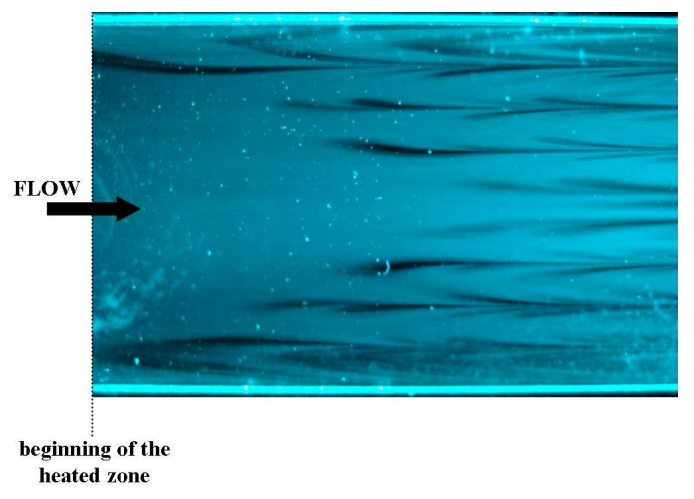

FIG. 1. Top view of the flow pattern in the entrance zone of the experimental air channel used in this study. The photo shows the mark of the injected oil particles in the horizontal laser sheet at channel mid-height. Longitudinal rolls develop from the sidewalls at the beginning of the heated zone then propagate to the center and spread over the entire width of the domain at a distance, $L_{\text {entrance }}$, from the inlet.

Note that a slightly different initiation mechanism of the longitudinal rolls has also been observed in channels heated from below, but at constant heat flux and at (very) high Rayleigh numbers, by ${ }^{17}$ and ${ }^{18}$ in water and by ${ }^{15}$ in air. Thermal plumes are shown to appear in the channel core just downstream the entrance and to superpose to one or two longitudinal rolls nearby each side wall.

To our knowledge, very few studies have been carried out to highlight the effects of the sidewalls on the onset and development of the longitudinal rolls. The numerical work of ${ }^{19}$ mainly focuses on the influence of the entrance temperature on the length of the developing zone and briefly mentions the influence of thermal conditions at the sidewalls on the flow 
behavior for a fixed transverse aspect ratio value. ${ }^{12}$ studied experimentally the influence of the transverse aspect ratio effect on the flow structure. Unfortunately, in this work as in most experimental studies reported in the literature, the longitudinal dimension of the heated zone is so small that developing rolls spread over the entire length of the test section and fully established longitudinal rolls are rarely observed. It is indeed known that the entrance length, $L_{\text {entrance}}$, for the full development of steady longitudinal rolls increases with increasing Reynolds number or decreasing Rayleigh number, i.e. with decreasing the buoyancy to inertia ratio (Richardson number) $R i=\operatorname{Ra} /\left(\operatorname{Pr} R e^{2}\right)\left({ }^{14},{ }^{20},{ }^{21}\right)$. Consequently, observing the development of rolls in too short channels led previous investigator to consider large values of $R i$, with the drawback that unsteady patterns readily develop, impairing a detailed analysis of the longitudinal rolls development $\left({ }^{12},{ }^{13}\right)$.

Among the few experiments with longitudinal extent suitable for the observation of fully developed steady longitudinal rolls, little were interested in the spatial distribution of the pattern. ${ }^{22}$ found that the average wavelength of the rolls was approximately equal to twice the height of the layer for large aspect ratios even for Rayleigh numbers far above the critical value thus confirming the early analytical and experimental study of ${ }^{23}$. This property can be also detected in the studies of ${ }^{24},{ }^{25}$ or $^{26}$. Another result which can be inferred from all numerical and experimental studies available in the literature is that the number of rolls is always even whatever the Rayleigh and Reynolds numbers and the transverse aspect ratio value. However, mechanisms of roll splitting and merging reported in the experiments of ${ }^{13}$ are not well understood. Moreover an experimental study on the stability of the longitudinal rolls performed by ${ }^{21}$ showed a great sensitivity of the number of longitudinal rolls to the experimental protocol which thus requires further investigation.

The aim of this paper is to investigate the effect of the transverse aspect ratio and the thermal conditions at the entrance and on the vertical and top walls of the channel on the onset and development of the steady longitudinal rolls. A combined experimental and numerical approach is used. The experimental setup used in this study is characterized by a heated zone of large longitudinal extent. This allows us to observe fully developed longitudinal rolls in a wide range of Reynolds and Rayleigh numbers $(3500 \leq R a \leq 6000$ and $20 \leq R e \leq 200)$. The $(R e, R a)$ combination is selected so as to generate steady patterns that typically correspond to $R i<7$. For larger values of $R i$, thermal plumes develop in the entrance zone of the channel leading to complex unsteady patterns downstream. 
Visualizations are carried out to study time and spatial evolutions of the flow pattern for the transverse aspect ratio ranging from 4 to 11 and the entrance temperature varying from the cold temperature of the upper plate to the hot temperature of the bottom one. Threedimensional numerical simulations are performed in a larger range of aspect ratios (from 4 to 18.4) to improve the understanding of the phenomena observed in the experiments and to provide information on the thermal and dynamic fields. In particular, numerical simulations allow us to investigate the influence of the thermal boundary conditions on the lateral and top walls on the flow development and help us to clarify the experimental observations.

The paper is organized in the following manner. After the presentation of the experimental facility and procedure in $\S 2$ and the numerical codes in $\S 3$, the results are discussed in $\S 4$. First the spatial and temporal evolution of the experimental flow is described. Then, the influence of the aspect ratio and of the thermal boundary conditions at the walls are discussed and finally the effect of the inlet temperature on the final wavenumber is studied. Concluding remarks are given in $\S 5$.

\section{EXPERIMENTAL APPARATUS AND PROCEDURE}

The experimental setup used in this study is a modification to that described in detail in ${ }^{21}$. A sketch of the apparatus is shown in figure 2. An air flow is introduced in a rectangular channel heated from below and cooled from above at constant temperatures, $\mathrm{T}_{\mathrm{h}}$ and $\mathrm{T}_{\mathrm{c}}$ respectively. The channel is on a vibration control system and the whole experimental setup is enclosed in a thermally regulated room maintained at a constant temperature, $\mathrm{T}_{\text {room }}$. The air flow rate is regulated using a flow controller located ahead of a settling chamber. The air flow enters the channel at $\mathrm{T}_{\text {room }}$ and the inlet temperature is then controlled by changing $\mathrm{T}_{\text {room }}$.

The channel is divided into three parts: an unheated zone of $50 \mathrm{~cm}$ length located upstream the heated zone allowing for the development of the Poiseuille flow, the heated region then an unheated zone of $40 \mathrm{~cm}$ placed downstream to minimize exit effects. The length of the heated region is $L=2 \mathrm{~m}$, designed so as to allow for a fully developed vortex flow for a large range of parameters, $30 \leq R e \leq 300$ and $R a \leq 12000$. The lower plate of the test section is made of a $10 \mathrm{~mm}$ thick and $175 \mathrm{~mm}$ width copper plate maintained at a constant temperature using five independent electric heaters. The upper plate of the test 


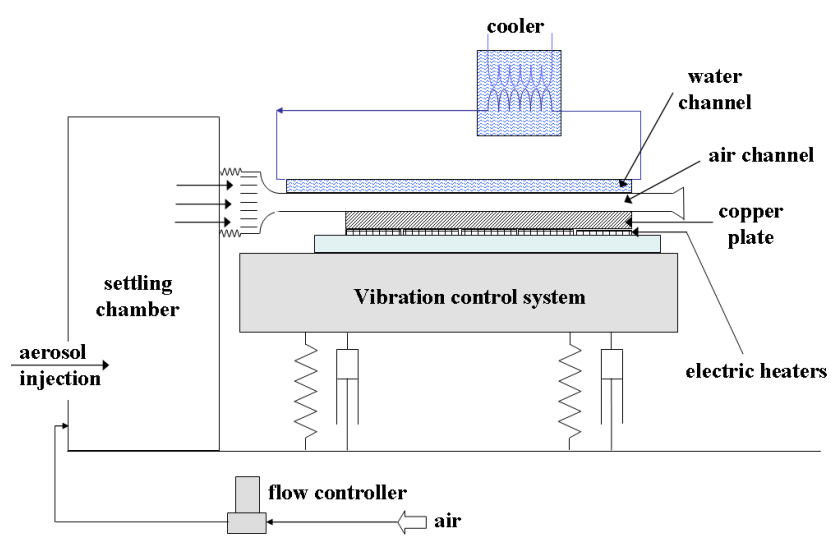

FIG. 2. Sketch of the experimental set-up.

section is made of $5 \mathrm{~mm}$ thick polycarbonate sheet maintained at a constant temperature using water circulation in a channel above it. For the range of temperature considered in this paper, the relative error on the Rayleigh number value is estimated to be less than 8 percent. The detailed analysis is available $i^{21}$. The lateral walls of the channel are made of polycarbonate moving plates allowing for a continuous change in the width of the air channel, from zero to $160 \mathrm{~mm}$. Three different heights of plates are available: $H=10 \mathrm{~mm}$ or $15 \mathrm{~mm}$ or $20 \mathrm{~mm}$. Depending on the combination between height and spacing, the range of transverse aspect ratios investigated in this paper is $4 \leq B \leq 12$. The spacing between plates is first gauged using calibrated wedges then a precise adjustment is performed by using a camera to obtain a spacing variation less than $1 \mathrm{~mm}$ on the entire length of the channel. The position of the side walls is monitored during experiments. The resulting relative error on the aspect ratio value is estimated to be less than 6 percent. Flow visualizations are conducted by using a $1 \mathrm{~mm}$ thick plane light beam together with oil particles introduced in the settling chamber upstream from the channel. The laser sheet is located either horizontally at midheight of the channel to observe the patterns from the top or vertically at various longitudinal locations allowing for a cross-sectional view of the rolls.

Standard experiments started from an initial isothermal Poiseuille flow for which all the system (bottom and upper walls, lateral walls and the air flow) was maintained at the cold temperature. At time time $=0 s$, the bottom plate was heated and the stationary state of the hot plate was achieved at a time $t_{\text {stat }}$. An example of a typical hot temperature ramp is 


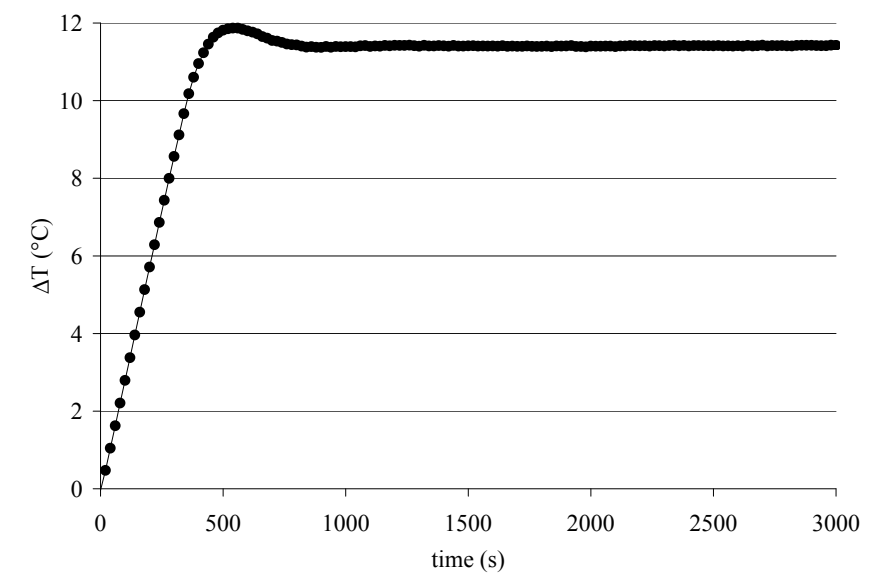

FIG. 3. Time evolution of the bottom plate temperature in the experiments. $B=5, R a=4500$, $R e=60$.

illustrated on Figure 3 for $R e=60$ and $R a=4500$. Here $t_{\text {stat }}$ is about $800 \mathrm{~s}$, with a weak overshoot around $500 s$.

The transient evolution of the pattern was visualized from the initial to the stationary state of the system. In order to check that the asymptotic flow pattern was independent of the initial thermal conditions, some experiments were carried out starting from a RayleighBénard configuration $(R e=0)$ for which the initial pattern corresponds to convective rolls perpendicular to the axis of the channel. The flow rate was then imposed at the desired Reynolds number. In this case, a long time was required to stabilize the flow, mainly due to the initial thermal print in the lateral walls, but the final pattern is the same as the previous case. It was thus concluded that the asymptotic state was independent of the initial condition.

\section{NUMERICAL PROCEDURE}

The numerical study has been carried out using two different codes. The first one is our own laboratory code specially developed to simulate the time evolution of RBP flows in rectangular channels with thin walls. That is the heat conduction in thick walls cannot be simulated. In this code, the unsteady three-dimensional Navier-Stokes and energy equations under the Boussinesq assumption are solved using a finite difference method, optimized for vectorial computers. The time discretization scheme is a second-order Adams-Bashforth scheme. The equations are discretized in space on uniform, Cartesian and staggered grids 


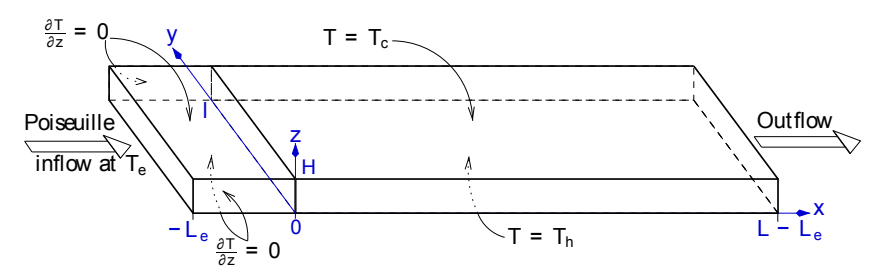

FIG. 4. Geometry and inlet, top and bottom thermal boundary conditions. The vertical lateral walls between $x=0$ and $x=A-A_{e}$ can be adiabatic, isothermal at $T_{h}$ or purely conductive (linear temperature vertical profile).

using second-order centered differences for the diffusive terms and a second-order central scheme for the convective terms. The time integration and the velocity-pressure coupling are computed by the projection method based on Goda's algorithm ${ }^{27}$. The Helmholtz and Poisson equations resulting from the space and time discretizations are solved by direct factorization methods based on the highly vectorizable TDMA algorithm. Steady state solutions are obtained by integrating long enough. A detailed description of this code, of its performances and of several validation tests are presented in ${ }^{28}$.

The computational domain used with this code is a horizontal rectangular channel of length L, width $\mathrm{W}$ and height $\mathrm{H}$ (see Figure 4). The four walls parallel to the channel axis are impermeable and no slipping. After an adiabatic entrance zone of length $\mathrm{L}_{\mathrm{e}}$, the top and bottom walls are kept at temperatures $T_{h}$ and $T_{c}$ respectively, with $T_{h}>T_{c}$. The thermal boundary conditions imposed at the vertical side walls at $x>0$ can be either of the Neumann or the Dirichlet type: adiabatic and purely conductive (linear temperature profile: $\left.T=T_{h}+\left(T_{c}-T_{h}\right) z / H\right)$ boundary conditions have been tested. A fully developed three-dimensional Poiseuille flow, characterized by a mean velocity $\mathrm{U}_{\text {mean }}$ and a constant temperature $\mathrm{T}_{\mathrm{e}}$, is imposed at inlet and an Orlanski-type boundary condition is imposed at outlet. With the origin of the coordinate system being placed at the beginning of the heated plate, the computational domain is defined by $(\mathrm{X}, \mathrm{Y}, \mathrm{Z}) \in\left[-A_{e}, A-A_{e}\right] \times[0, B] \times[0,1]$ in dimensionless Cartesian coordinates, where $A=\mathrm{L} / \mathrm{H}, B=\mathrm{W} / \mathrm{H}$ and $A_{e}=\mathrm{L}_{\mathrm{e}} / \mathrm{H}$.

We denote by $\theta=\left(\mathrm{T}-\mathrm{T}_{\mathrm{c}}\right) /\left(\mathrm{T}_{\mathrm{h}}-\mathrm{T}_{\mathrm{c}}\right)$ the dimensionless reduced temperature. At the initial time step $(t=0)$, the inlet fully developed and isothermal Poiseuille flow, at $\theta_{e}=$ $\left(\mathrm{T}_{\mathrm{e}}-\mathrm{T}_{\mathrm{c}}\right) /\left(\mathrm{T}_{\mathrm{h}}-\mathrm{T}_{\mathrm{c}}\right)$, is imposed in all the channel. For $t>0$ and $X>0$, the heating $\left(\theta_{h}=1\right)$ is imposed on the lower horizontal wall while the upper wall is cooled $\left(\theta_{c}=0\right)$. The four walls of the upstream zone $(X<0)$ are always kept adiabatic. It has been verified that 
the number of rolls at the asymptotic steady-state is independent of the initial conditions.

Preliminary calculations showed that the Reynolds number had no influence on the properties of the fully-developped longitudinal rolls in the range $30 \leq R e \leq 200$. To be close to the experiments, the Reynolds and Prandtl number are fixed at $R e=40$ and $\operatorname{Pr}=0.71$. The longitudinal aspect ratio $A$ vary between 50 and 100 and the entrance aspect ratio is fixed at $A_{e}=3$ or 5 . No influence of these aspect ratios was observed on the longitudinal roll development. The ranges of variation of the main adjustable parameters of the code were $3500 \leq R a \leq 6000,4 \leq B \leq 20$ and $0 \leq \theta_{e} \leq 1.2$ for all the simulations of the present work. These simulations have been carried out with the dimensionless time step $\Delta t=0.02$ and with two uniform grids of dimensionless sizes $\Delta X \times \Delta Y \times \Delta Z=0.2 \times 0.08 \times 0.025$ and/or $0.15 \times 0.043 \times 0.025$ depending on the cases. The same transitions of the total number of rolls with $B$ were found with these two grids, and also with a much finer grid $\Delta X \times \Delta Y \times \Delta Z=0.11 \times 0.026 \times 0.02$ that was used only to test the space convergence of the solutions for four different values of the aspect ratio $B$.

The second code used to take into account the heat conduction in the channel walls was the commercial code Ansys/Fluent ${ }^{29}$. To reduce the computational costs, steady simulations of the longitudinal rolls were performed by taking into account the symmetry through the vertical mid-plane at $y=l / 2$. This code is a colocated finite volume code. To solve the incompressible Navier-Stokes and energy equations with the Boussinesq approximation and to deal with the velocity/pressure coupling, we used SIMPLEC algorithm and Quick scheme for the discretization of the convective and diffusive fluxes. The steady simulations have been continued until the scaled residuals reach values less than $10^{-8}$ for all the solved equations.

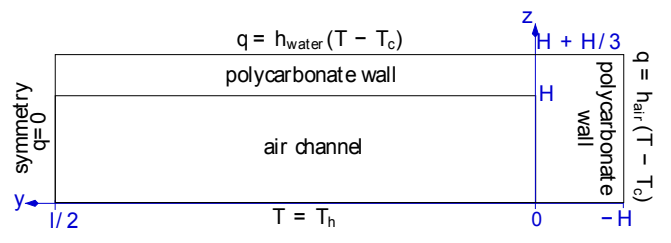

FIG. 5. Cross section of the computational domain and thermal boundary conditions when the symmetry through the vertical mid-plane and the heat conduction in the vertical and top walls of the channel are taken into account.

A cross section of the rectangular computational domain used to simulate the experimental channel is presented in Figure 5 . The horizontal bottom wall is heated at $T=T_{h}$. The 
top and vertical right walls are polycarbonate walls of thicknesses $H / 3$ and $H$ respectively, with $H=1.5 \mathrm{~cm}$. In the experimental apparatus, the outer boundary of the top wall is in contact with the cooling water channel. Thus a convective heat transfer with water at $T=T_{c}$ and a heat transfer coefficient $h_{\text {water }}=500 \mathrm{~W} / \mathrm{m}^{2} \mathrm{~K}$ is imposed as boundary condition. As the external face of the vertical wall is in contact with room air at rest in the experiments, a convective transfer with air at $T=T_{c}$ and a heat transfer coefficient $h_{\text {air }}=4 \mathrm{~W} / \mathrm{m}^{2} \mathrm{~K}$ is imposed as boundary condition.

Two simulation series have been performed with this code: the first one for $B \approx 2.4$ to observe the transition between four and six longitudinal rolls and the second one for $B \approx 5.3$ to observe the transition between ten and twelve rolls. In the two series, the longitudinal and entrance aspect ratios of the channel are respectively equal to $A=50$ and $A_{e}=3$. The hexahedral mesh used is uniform and the dimensionless cell size is $\Delta X \times \Delta Y \times \Delta Z=$ $0.167 \times 0.052 \times 0.028$. We fixed $T_{c}=293 K$ and $T_{h}=309 K$. The densities, thermal conductivities and specific heat of air and polycarbonate and the dynamic viscosity of air were fixed at $\rho_{\text {air }}=1.18 \mathrm{~kg} / \mathrm{m}^{3}, \rho_{\text {polyc }}=1200 \mathrm{~kg} / \mathrm{m}^{3}, k_{\text {air }}=0.02645 \mathrm{~W} / \mathrm{mK}, k_{\text {polyc }}=$ $0.19 \mathrm{~W} / \mathrm{mK}, C p_{\text {air }}=1005 \mathrm{~W} / \mathrm{mK}, C_{\text {polyc }}=1200 \mathrm{~W} / \mathrm{mK}$ and $\mu_{\text {air }}=1.8526 \times 10^{-5} \mathrm{~kg} / \mathrm{ms}$. The Reynolds and Prandtl numbers were equal to $R e=40$ and $\operatorname{Pr}=0.7$ and the Rayleigh number, computed using the average temperature of the top plate of the air channel was approximately equal to $R a \approx 4400$.

\section{RESULTS}

\section{A. Temporal and spatial evolution of the longitudinal rolls}

Starting from a cold isothermal initial state, a typical scenario of the complete experimental transient flow behavior is illustrated in figures 6 and 7 . The flow is visualized in a cross section located at a distance $x=165 \mathrm{~cm}(X=110)$ from the start of the heated zone. The scenario can be divided into two stages. The first stage (figure 6 ) is very similar for all experiments. A few seconds after the heating starts, rolls first appear nearby the lateral walls and then develop sequentially by pair towards the center of the channel. The apparatus is such that the conducting sidewalls are in contact with the bottom plate and thus gradually heat up as the bottom plate is heated. Due to the large thermal inertia of 

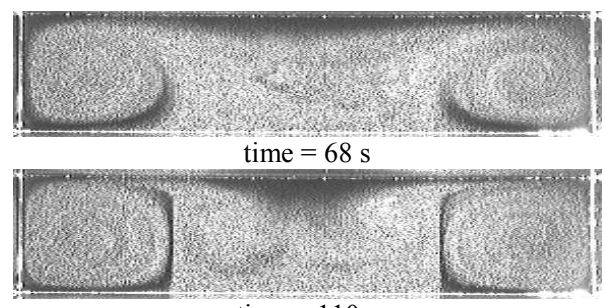

time $=110 \mathrm{~s}$

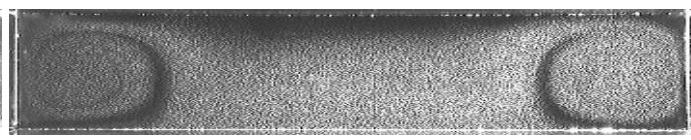

time $=68 \mathrm{~s}$

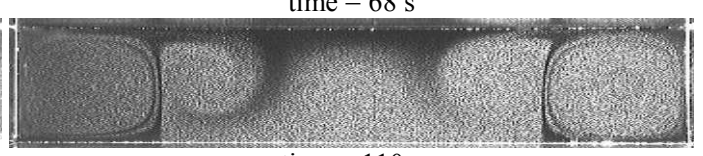

time $=110 \mathrm{~s}$

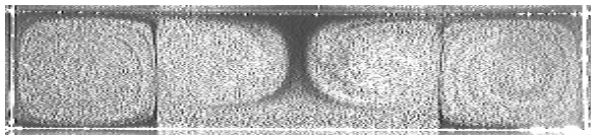

time $=140 \mathrm{~s}$

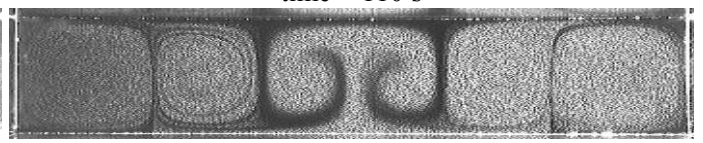

time $=140 \mathrm{~s}$

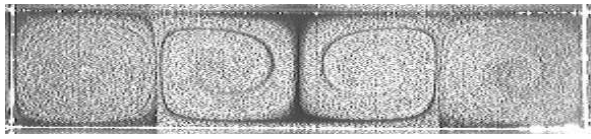

time $=166 \mathrm{~s}$

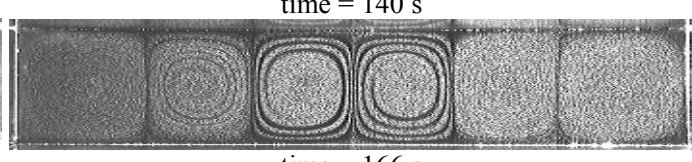

time $=166 \mathrm{~s}$

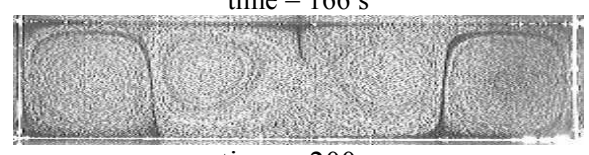

time $=200 \mathrm{~s}$
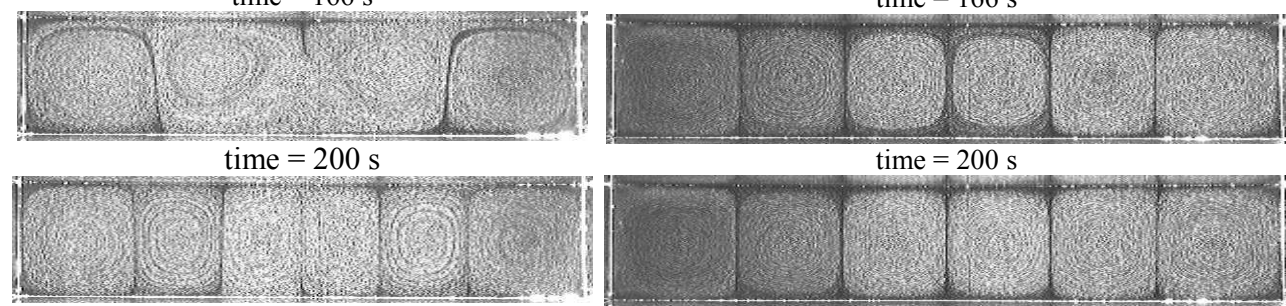

time $=252 \mathrm{~s}$

(a) $B=5, R e=60\left(\mathrm{U}_{\text {mean }}=5 \mathrm{~cm} / \mathrm{s}\right), R a=4500$

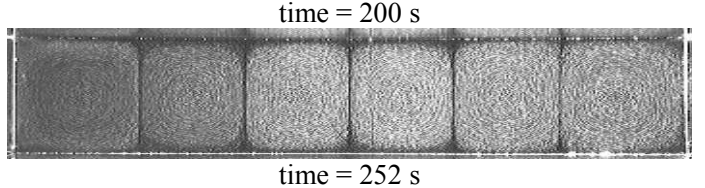

(b) $B=6, R e=42\left(\mathrm{U}_{\text {mean }}=4 \mathrm{~cm} / \mathrm{s}\right), R a=4500$

FIG. 6. Transient behavior of the flow during the first stage of development of the rolls. Crosssectional view at $x=165 \mathrm{~cm}(X=110)$. Initially all the system is maintained at the cold temperature, $\theta_{c}=0$. At time $=0$ the bottom plate is heated and the inlet temperature is maintained at $\theta_{e}=0$. The rolls develop sequentially from the sidewalls to the center of the channel with descending end-rolls along the vertical walls.

the polycarbonate compared with air (the thermal diffusivity of air is 150 times greater than that of polycarbonate), the sidewalls remain much colder than the adjacent heated flow and a resulting downward end-roll pattern is observed. At the end of this stage, the two cases presented in figures $6(\mathrm{a})$ and (b) for aspect ratios $B=5$ and $B=6$ are characterized by a 6-cell mode that clearly differs as for the size of the central pair.

The second stage of development is displayed in figure 7 and is related to the thermal evolution of the lateral walls toward an equilibrium state. Starting from a N-cell mode, a cellular flow adjustment is observed which depends on the aspect ratio value. For odd values, the descending end-rolls weaken as the sidewalls are gradually heated until their complete extinction, resulting in a $(N-2)$-cell mode with ascending end-rolls (see $7(\mathrm{a}))$. 


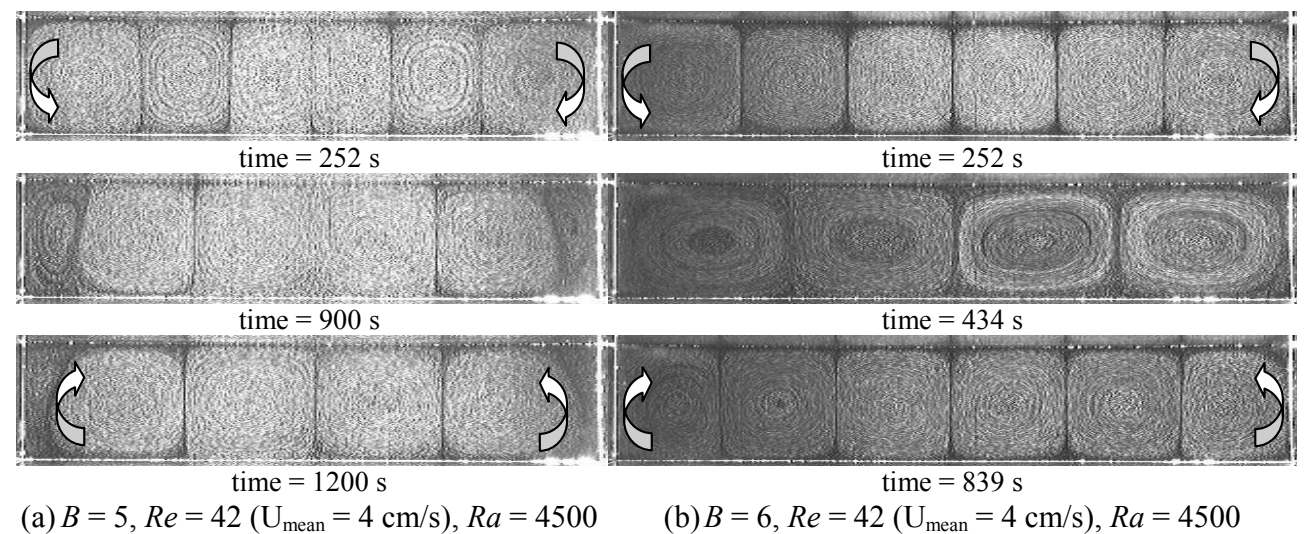

FIG. 7. Transient behavior of the flow during the second stage of development of the rolls following the first stage displayed in figure 6 . Cross-sectional view at $x=165 \mathrm{~cm}(X=110)$. An adjustment in the cell arrangement is observed toward a steady state characterized by an even number of rolls with ascending end-rolls along the vertical walls.

For even values of the aspect ratio the flow adjustment is very chaotic with cells merging or splitting and with transient $(N-2)$-cell modes (see 7(b)). At steady state, a $N$-cell mode pattern is observed with an upward motion nearby the sidewalls. These scenarios are perfectly reproducible and were observed for all the experiments performed with various values of the aspect ratio. The resulting flow structure consists in a symmetric pattern of convection rolls ascending along the lateral walls at steady-state, whatever the aspect ratio. This is in agreement with the experimental results of ${ }^{26}$ or $^{13}$ among others.

In the numerical simulations the horizontal walls are brought to their final steady temperature instantaneously and the boundary conditions on the lateral walls are time independent. Longitudinal rolls are initiated by pair from the very beginning of the heated zone and then propagate downstream at the mean flow velocity. For all the types of boundary conditions applied at the sidewalls, an upward motion along these walls is observed when the inlet temperature is equal to zero. This point will be discussed further. The characteristic time of the transient stage is directly related to the mean flow velocity, hence to the Reynolds number. This time is typically $50 \mathrm{~s}$ in the numerical simulations for a $2 \mathrm{~m}$ long channel for the set of parameters corresponding to figures 6 and 7, to compare with the experimental transient time of about $1200 \mathrm{~s}$ corresponding to the conducting timescale of the sidewalls $\left(\approx H^{2} / \kappa_{\text {polyc }}\right)$. 


\section{B. Effect of aspect ratio on the number of rolls}

We now focus our attention on the aspect ratio effect on the number of rolls, $N$, at steady state and for the inlet temperature $\theta_{e}=0$. In agreement with the experiments, simulations always lead to an even number of rolls and $N$ increases as $B$ increases. Moreover, $N$ was found to be independent of $R e$ and $R a$ in the range under study (3500 $\leq R a \leq 6000$ and $20 \leq R e \leq 200)$. All the results presented below and in the next section refer to the case $R a=4500$ and $R e=40$.

The critical values of the aspect ratio for which a change in the number of rolls is detected are reported in table I related to the experiments and to the simulations conducted with adiabatic sidewalls or with a linear temperature distribution at the sidewalls.

\begin{tabular}{|c||c|c|c|}
\cline { 2 - 4 } \multicolumn{1}{c|}{} & \multicolumn{2}{c|}{ Numerical simulations } & Experiments \\
\hline$N$ & adiabatic sidewalls & linear distribution & conducting walls \\
\hline \hline $4-6$ & 4.8 & 4.8 & 5.3 \\
\hline $6-8$ & 6.6 & 6.6 & 6.9 \\
\hline $8-10$ & 8.5 & 8.6 & 8.9 \\
\hline $10-12$ & 10.4 & 10.5 & 10.8 \\
\hline $12-14$ & 12.4 & 12.5 & 13 \\
\hline
\end{tabular}

TABLE I. Values of the aspect ratio shortly before the change in the number of rolls, $B_{\max }$, for $\theta_{e}=0, R a=4500, R e=40$.

The transition occurs systematically later in the experiments than in the simulations. On the other hand, the critical values are quite similar for the adiabatic condition and the linear distribution which means that the thermal state of the lateral walls does not significantly affect the transition.

A simulation has been carried out using Ansys/Fluent software to account for the heat conduction through the thickness of the sidewalls so as to be closer to realistic thermal boundary conditions. Figure 8 displays the resulting temperature profile at one of the sidewalls (at $y=0$, see figure 5 ) for $B=10.4$, compared with the corresponding previous numerical results obtained using the other two sidewall boundary conditions. We notice 
that the thermal equilibrium of the conducting sidewalls almost corresponds to a linear temperature distribution. Accordingly, we assume that this thermal state prevails at the vertical walls in our experimental channel.

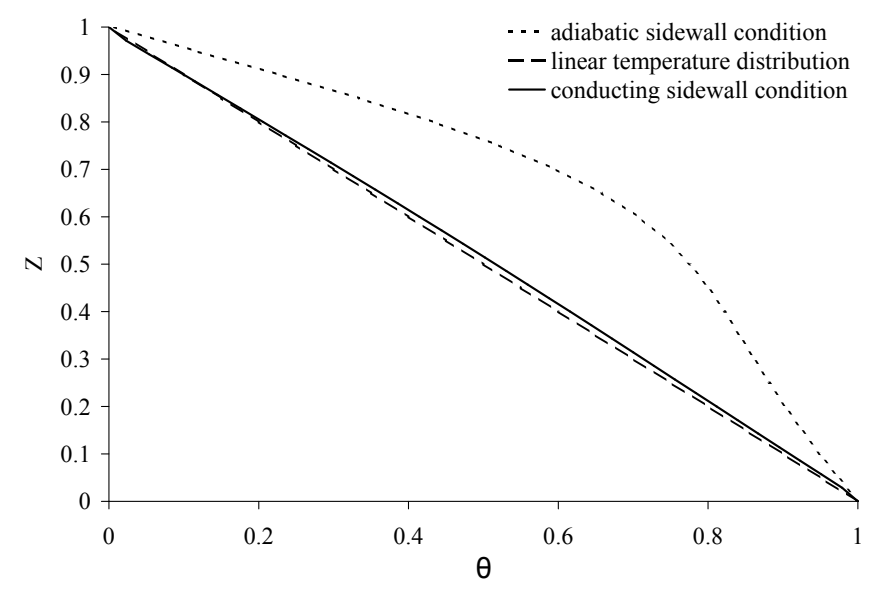

FIG. 8. Temperature profiles at the sidewalls - Numerical simulations - $R e=40, R a=4500$, $\theta_{e}=0, B=10$.

The discrepancy between experimental and numerical transitions obtained with a linear temperature distribution could be related to the effect of the finite thermal conductivity of the upper boundary in the experiments. This assumption is based on a result reported in ${ }^{30}$ as follows: in Rayleigh-Bénard convection, the wavelength of the convection rolls becomes larger with decreasing the ratio $R=k_{\text {wall }} / k_{\text {fluid }}$ between the thermal conductivity of the horizontal walls and that of the fluid. Note that in our experiments the conductivity of the polycarbonate used to design the lateral and top walls is 7 times greater than that of the air flowing in the channel. To validate this feature in our RBP configuration, numerical steady simulations have been performed using Ansys/Fluent for two transitions (4-6 and 10-12), by taking conducting boundary conditions at the lateral and top walls similar to those in our experiments (see end of section III). The resulting thermal field in a cross section for the $10-12$ transition is displayed in figure 9. The deviation of the isotherms occurs not only in the fluid layer as in the infinitely conducting case but also inside the thickness of the top wall leading to an increase in the wavelength of the rolls as described $i^{30}$ in Rayleigh-Bénard convection. The critical aspect ratios shortly before the change in the number of rolls are now equal to 5 for the $4-6$ transition ( $-6 \%$ compared to the experiments) and 10.7 for the $10-12$ transition (less than 1\% compared to the experiments). 


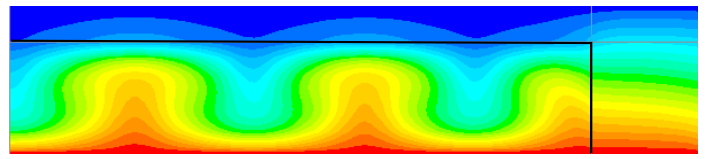

FIG. 9. Cross section of the temperature field in the half-width of the channel at $x=65 \mathrm{~cm}$ (see Figure 5). Fluent simulations performed with finitely conducting top and lateral boundaries: $R=k_{\text {wall }} / k_{\text {air }}=7-B=10.6, R e=40, R a \approx 4500, \theta_{e}=0$.

An example of the steady-state flow structure in the form of temperature fields obtained in the simulations just before and just after the change in the number of rolls is displayed in Figure 10. The onset of an additional pair of rolls always occurs in the center of the channel and the flow along the longitudinal central axis is alternatively upward or downward from a transition to the next transition.

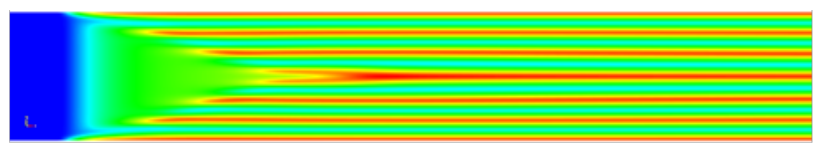

(a) $\mathrm{B}=12.4 ; \mathrm{N}=12$

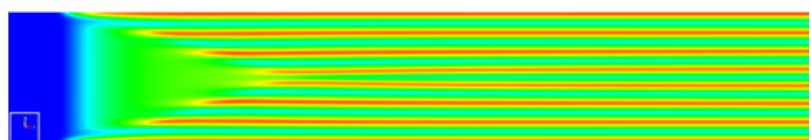

(b) $\mathrm{B}=12.5 ; \mathrm{N}=14$

FIG. 10. Horizontal temperature fields at $Z=0.5$ for $B$ shortly before and shortly after the onset of an additional pair of rolls - Adiabatic case - $R e=40, R a=4500, \theta_{e}=0$.

It is worthwhile to note that the longitudinal rolls at steady state are always ascending along the lateral walls, not only in the experiments where thermal effects due to not perfectly conducting walls may be responsible for this result, but even in the $3 \mathrm{D}$ numerical simulations performed with adiabatic sidewall conditions and a cold inlet temperature (Figure 10). This feature is also observed in the studies of ${ }^{13},{ }^{2},{ }^{19}$ and $^{31}$ among others. Note that this upward motion explains why the wall is hotter than the average in the adiabatic case (see figure 8). We are going to show that this behavior is due to the presence of the velocity boundary layers along the lateral walls. Indeed, at a given height, $Z$, in the channel, a particle of fluid inside the velocity boundary layer nearby the lateral walls moves more slowly than a particle located outside the boundary layer. Consequently at a given streamwise location, $X$, just downstream the entrance of the heated zone, the fluid flowing in the region adjacent to the sidewalls is warmer and thus less dense than the fluid in the center part of the channel since the inlet temperature is cold. The spanwise density gradient gives rise to an ascending motion nearby the sidewalls at the beginning of the channel, which propagates downstream 
leading to ascending longitudinal rolls on the entire length of the domain.

The main result pointed out by the previous analysis is that a combined spatial and temporal approach is required to understand the behavior of the longitudinal rolls in the channel. The initiation mechanism is symmetric with regard to the central axis resulting inevitably in an even number of rolls. As a consequence, the allowed band of wavenumbers for the longitudinal rolls in RBP flows is restricted compared with Rayleigh-Bénard convection in finite aspect ratio boxes, for which an integral number of rolls (odd or even) is theoretically expected. In addition, note that in the temporal linear stability analysis of longitudinal rolls performed in RBP flows in finite extension channels $\left({ }^{4,32}\right)$ the spatial development of the rolls was not taken into account and stable solution branches with odd or even number of rolls were detected.

The inner rolls gradually appear in the core by pair in the entrance zone with a size of order $H$ while at the same time they are advected by the flow at the mean velocity. Once all the rolls in place at the end of the entrance zone, an adjustment of the pattern is observed downstream. Experimental measurements have shown that in a first approximation, excluding the two end-rolls nearby the lateral walls, the size of the inner rolls is constant for a given $B$. The mean wavenumber values, $\alpha$, obtained in the experiments and in the simulations with adiabatic sidewalls performed are reported in Figure 11, for aspect ratios just before (black marks) and just after (white marks) each transition. These two series define the boundaries between respectively the smallest and the largest wavenumber that can be observed for a given $N$. For small values of $B$, the size of the inner rolls is strongly affected by the presence of the sidewalls and the onset of an additional pair of rolls results in a strong compression of the rolls in the channel core. For large values of $B$, the sequential process of formation of the pattern in the entrance zone forces the range of allowed wavelengths around $2 \mathrm{H}$, unlike in the Rayleigh-Bénard configuration for which an adjustment of the rolls to larger wavelengths is expected for the range of Rayleigh numbers investigated $\left(33,{ }^{34}\right)$. For the lower curve the wavenumber becomes constant for $B>10$, equal to 3.1. For the upper curve a variation of $\alpha$ is detected until $B \approx 14$. Then $\alpha$ reaches a constant value of 3.5 reflecting a weak compression of the pattern. These results suggest that the roll adjustment process is no longer affected by the presence of the sidewalls only for aspect ratios larger than 14 and in that case the range of permitted wavenumbers is very narrow : $3.1 \leq \alpha \leq 3.5$. 


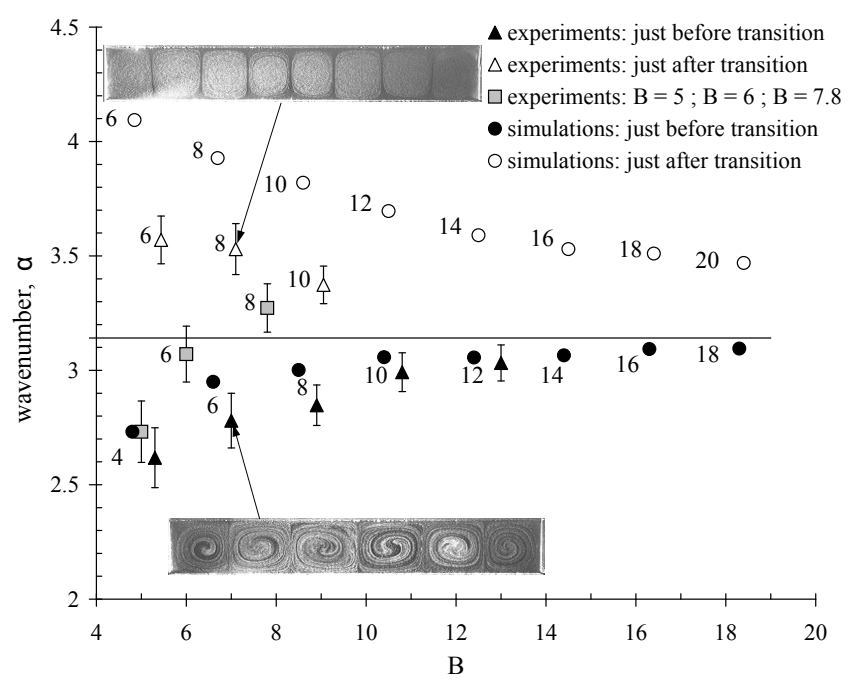

FIG. 11. Mean wavenumber, $\alpha$, related to the inner rolls as a function of $B$ in the fully developed steady state - The numbers refer to the values of $N$ - numerical simulations (adiabatic case) and experiments $-\theta_{e}=0, R e=40, R a=4500$.

\section{Effect of inlet temperature on the number of rolls}

During the course of the experimental study presented in the previous section, it was detected that small variations in the inlet temperature could affect the structure of the flow. An increase in $\theta_{e}$ results in the onset of an additional pair of rolls in the center of the channel in the entrance zone which then propagates downstream at the mean flow velocity. Fig 12 displays a top view of the flow during the change from a 8-cell mode to a 10-cell mode for $B=9$, following a change in $\theta_{e}$ from 0 to 0.1 . The system returns to its initial state if the inlet temperature is decreased to zero. This feature has been observed experimentally for values of $B$ close to the critical values detected in the previous section even for small variations of $\theta_{e}$.

Numerical simulations were carried out in the adiabatic case to further analyze this behavior. Figure 13 shows that the effect of the change in the inlet temperature is well reproduced by the simulations.

A numerical sequence of the onset of additional rolls is displayed in figure 14 in the adiabatic case. An increase in the inlet temperature results in a weakening of the spanwise temperature gradients in the lateral boundary layers at the entrance. As a consequence, the intensity of the buoyancy induced flow is reduced and the magnitude of the first rolls nearby 


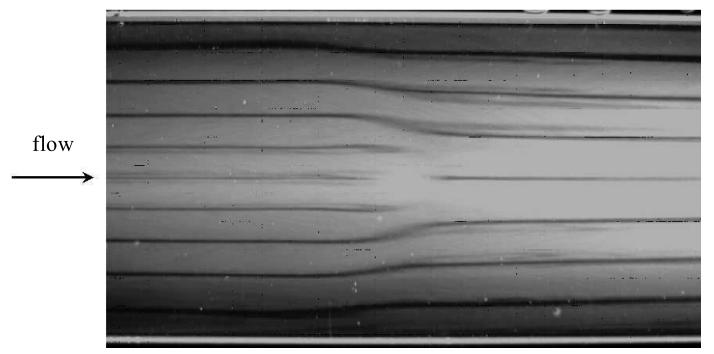

FIG. 12. Visualization of the experimental transition from 8 to 10 rolls following an increase in the inlet temperature from $\theta_{e}=0$ to $\theta_{e}=0.1$ - top view $-B=9, R e=50, R a=4500$.

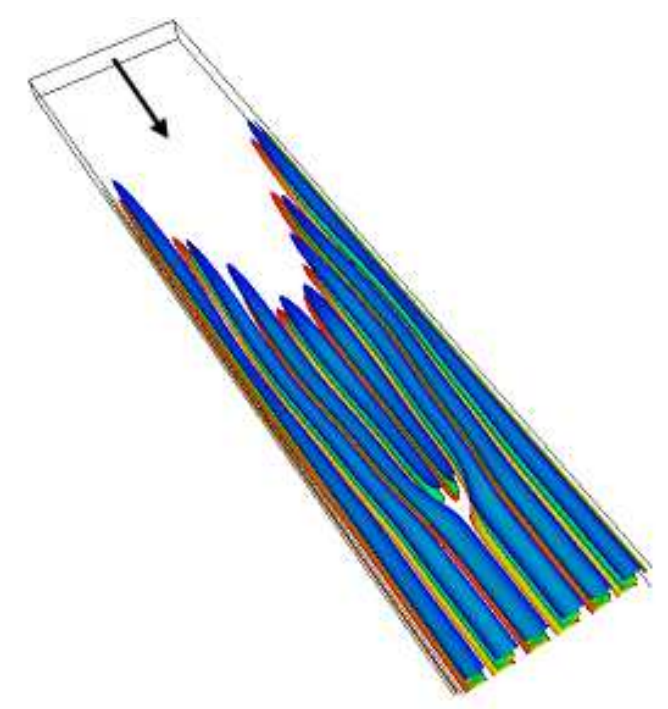

FIG. 13. Visualization in the form of 3D vorticity field of the onset of an additional pair of rolls following an increase in the inlet temperature from $\theta_{e}=0$ to $\theta_{e}=0.2$ - Numerical simulations $B=7, R e=40, R a=4500$.

the sidewalls in the entrance zone decreases. Thereby, the available space at the center of the channel expands, allowing for the growth of an additional pair of rolls. Once the new pair is triggered at the center, an adjustment of the roll size leads to a nearly uniform distribution over the width. Thus, transitions between a N-cell mode and a $(N+2)$-cell mode take place for smaller values of $B$ when $\theta_{e}$ is increased.

As long as the inlet temperature is less than 0.5, the flow is ascending along the sidewalls due to the initiation mechanism of the end rolls described in paragraph IV B. In the adiabatic case as well as if a linear temperature distribution is imposed at the sidewalls, an inversion in the rotation direction of the rolls nearby the sidewalls is observed for $\theta_{e}>0.5$, due to 
a similar but opposite process that gives rise to a downward motion inside the velocity boundary layers along the vertical walls at the beginning of the channel. This behavior was also detected in the experiments.

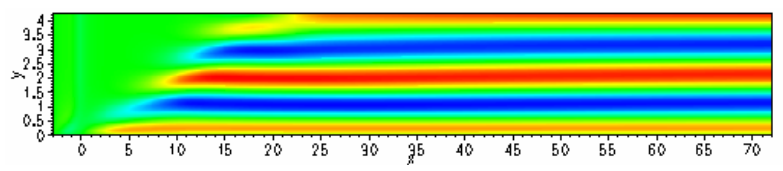

(a) $\mathrm{t}_{0}=0$ : initial time

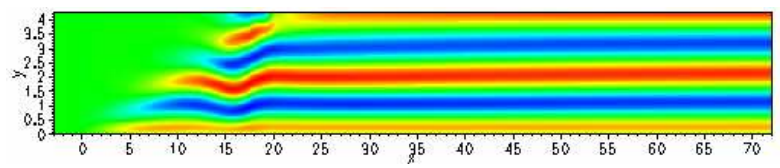

(c) $t_{2}=18$

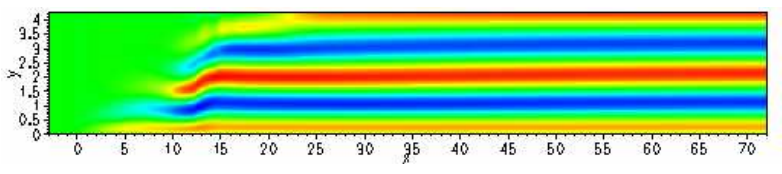

(b) $t_{1}=14$

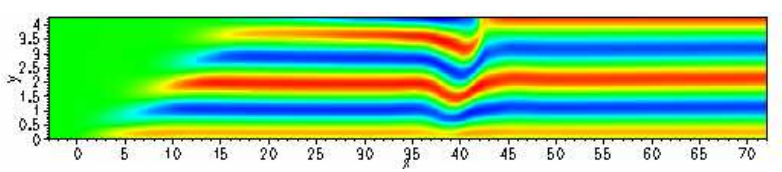

(d) $t_{3}=38$

FIG. 14. Time evolution of the horizontal temperature field at $Z=0.5$ in a mid-width of the channel for the adiabatic case - Effect of inlet temperature: starting from $\theta_{e}=0$ the inlet temperature is fixed at $\theta_{e}=0.2$ at $t_{0} . t_{1}$ and $t_{2}$ : the rolls nearby the sidewalls become smaller in the entrance zone allowing the onset of an additional pair in the available space at the center of the channel. $t_{3}:$ adjustment of the rolls $-B=8.5, R e=40, R a=4500$

\section{CONCLUSIONS}

The study presented in this paper has attempted to clarify the effects of aspect ratio and thermal conditions on the development of steady longitudinal rolls in a rectangular channel heated from below. Our main objective was to characterize the way to obtain reproducible and deterministic steady patterns resulting from this convective instability in order to later study precisely their stability.

After a transient phase that is clearly described in the paper, the asymptotic steady flow obtained experimentally is shown to be always ascending along the vertical side walls when inlet temperature is smaller than the average temperature of the channel $\left(T_{e}<0.5\right)$. It has been shown that the fully developed pattern in the form of longitudinal rolls is determined by the flow behavior in the entrance zone and more precisely by the properties of the first pair of rolls triggered at inlet nearby the sidewalls. The physical mechanism responsible for the development of these two longitudinal end-rolls is due to the presence of velocity boundary layers adjacent to the lateral walls. The inner rolls develop gradually towards the 
center of the channel while they are convected by the mean flow. The end-rolls are fixed at the lateral walls allowing the inner rolls to be maintained in the domain. The size of the two end-rolls is dependent on the inlet temperature and acts upon the final wavenumber observed in the asymptotic state for a given transverse aspect ratio. On the other hand, it is found that the heat conduction in the top horizontal wall of the experimental channel must be taken into account in the numerical simulations so that the experimental wavenumber can be properly depicted.

Finally, it has been shown that the roll size adjustment far from the entrance zone becomes independent of the aspect ratio for values larger than 14. In that case, the wavenumber varies inside a very narrow range of allowed values, $3.1 \leq \alpha \leq 3.5$.

Further studies are needed to investigate the influence of the Rayleigh number on the development of the longitudinal rolls. Particularly, for high aspect ratio values, it would be of interest to determine if critical conditions are required to observe rolls over all the width of the channel. Moreover, it would be interesting to study the influence of the aspect ratio on the behavior of the secondary wavy instability detected $i^{21}$, in particular to determine the effect on the spanwise magnitude of the oscillations within the framework of CVD applications for instance ${ }^{2}$.

\section{ACKNOWLEDGMENTS}

All the computations were carried out on the NEC-SX8 vectorial super computer at IDRIS-CNRS (Orsay, France) under research project 091474.

\section{REFERENCES}

${ }^{1}$ X. Nicolas, "Bibliographical review on the Poiseuille-Rayleigh-Bénard flows: the mixed convection flows in horizontal rectangular ducts heated from below," Int. J. Thermal Sciences. 41, 961-1016 (2002).

${ }^{2}$ X. Nicolas, A. Benzaoui, and S. Xin, "Numerical simulation of thermoconvective flows and more uniform depositions in a cold wall rectangular APCVD reactor," J. Crystal Growth 310, 174-186 (2008). 
${ }^{3}$ K. S. Gage and W. H. Reid, "The stability of thermally stratified plane Poiseuille flow," J. Fluid Mech. 33, 21-32 (1968).

${ }^{4}$ J.-M. Luijkx, J. K. Platten, and J. C. Legros, "On the existence of thermoconvective rolls, transverse to a superimposed mean Poiseuille flow," Int. J. Heat Mass Transfer 24, 1287-1291 (1981).

${ }^{5}$ M. T. Ouazzani, J. P. Caltagirone, G. Meyer, and A. Mojtabi, "Étude numérique et expérimentale de la convection mixte entre deux plans horizontaux à températures différentes," Int. J. Heat Mass Transfer 32, 261-269 (1989).

${ }^{6}$ M. T. Ouazzani, J. K. Platten, and A. Mojtabi, "Étude expérimentale de la convection mixte entre deux plans horizontaux à températures différentes -II," Int. J. Heat Mass Transfer 33, 1417-1427 (1990).

${ }^{7}$ J.-M. Luijkx, "Influence de la présence de parois latérales sur l'apparition de la convection libre, forcée et mixte," Thèse de doctorat, Université de Mons-Hainaut, Belgique (1983).

${ }^{8}$ X. Nicolas, J. M. Luijkx, and J. K. Platten, "Linear stability of mixed convection flows in horizontal rectangular channels of finite transversal extension heated from below," Int. J. Heat Mass Transfer 43, 589-610 (2000).

${ }^{9}$ H. W. Müller, M. Lücke, and M. Kamps, "Convective patterns in horizontal flow," Europhys. Lett. 10, 451-456 (1989).

${ }^{10}$ H. W. Müller, M. Lücke, and M. Kamps, "Transversal convection patterns in horizontal shear flow," Phys. Rev. A 45, 3714-3726 (1992).

${ }^{11} \mathrm{P}$. Carrière and P. A. Monkewitz, "Convective versus absolute instability in mixed Rayleigh-Bénard-Poiseuille convection," J. Fluid Mech. 384, 243-262 (1999).

${ }^{12}$ M. Y. Chang and T. F. Lin, "Experimental study of aspect ratio effects on longitudinal vortex flow in mixed convection of air in a rectangular duct," Int. J. Heat Mass Transfer 41, 719-733 (1998).

${ }^{13}$ M. Y. Chang, C. H. Yu, and T. F. Lin, "Changes of longitudinal vortex roll structure in a mixed convective air flow through a horizontal plane channel : an experimental study," Int. J. Heat Mass Transfer 40, 347-363 (1997).

${ }^{14}$ K.-C. Chiu and F. Rosenberger, "Mixed convection between horizontal plates -I. Entrance effects," Int. J. Heat Mass Transfer 30, 1645-1654 (1987).

${ }^{15}$ C. Gau, S. W. Liu, T. M. Huang, and W. Aung, "Secondary flow and enhancement of heat transfer in horizontal parallel-plate and convergent channels heating from below," 
Int. J. Heat Mass Transfer 42, 2629-2647 (1999).

${ }^{16} \mathrm{~J}$. R. Maughan and F. P. Incropera, "Regions of heat transfer enhancement for laminar mixed convection in a parallel plate channel," Int. J. Heat Mass Transfer 33, 555-570 (1990).

${ }^{17}$ F. Incropera, A. Knox, and J. Maughan, "Mixed convection flow and heat transfer in the entry region of a horizontal rectangular channel," J. Heat Transf. - Trans. of the ASME 109, 434-439 (1987).

${ }^{18}$ A. Benderradji, A. Haddad, R. Taher, M. Medale, C. Abid, and F. Papini, "Characterization of fluid flow patterns and heat transfer in horizontal channel mixed convection," Heat Mass Transf. 44, 1465-1476 (2008).

${ }^{19}$ U. Narusawa, "Numerical analysis of mixed convection at the entrance region of a rectangular duct heated from below," Int. J. Heat Mass Transfer 36, 2375-2384 (1993).

${ }^{20}$ Y. Kamotani and S. Ostrach, "Effect of thermal instability on thermally developing laminar channel flow," J. Heat Transfer 98, 62-66 (1976).

${ }^{21}$ H. Pabiou, S. Mergui, and C. Bénard, "Wavy secondary instability of longitudinals rolls in Rayleigh-Bénard-Poiseuille flows," J. Fluid Mech. 542, 175-194 (2005).

${ }^{22} \mathrm{~S}$. Ostrach and Y. Kamotani, "Heat transfer augmentation in laminar fully developed channel flow by means of heating from below," J. Heat Transfer 95, 220-225 (1975).

${ }^{23}$ Y. Mori and Y. Uchida, "Forced convective heat transfer between horizontal flat plates," Int. J. Heat Mass Transfer 9, 803-817 (1966).

${ }^{24}$ M. Akiyama, G. J. Hwang, and K. C. Cheng, "Experiments on the onset of longitudinal vortices in laminar forced convection between horizontal plates," J. Heat Transfer Ser. C 93, 335-341 (1971).

${ }^{25}$ K. Fukui, M. Nakajima, and H. Ueda, "The longitudinal vortex and its effects on the transport processes in combined free and forced laminar convection between horizontal and inclined parallel plates," Int. J. Heat Mass Transfer 26, 109-120 (1983).

${ }^{26}$ K.-C. Chiu, J. Ouazzani, and F. Rosenberger, "Mixed convection between horizontal plates -II. Fully developed flow," Int. J. Heat Mass Transfer 30, 1655-1662 (1987).

${ }^{27} \mathrm{~K}$. Goda, "A multistep technique with implicit difference schemes for calculating two-orthree-dimensional cavity flows," J. Comput. Phys. 30, 76-95 (1979).

${ }^{28}$ A. Benzaoui, X. Nicolas, and S. Xin, "Efficient vectorized finite difference method to solve the incompressible Navier-Stokes equations for 3d mixed convection flows in high aspect 
ratio channels," Num. Heat Transf. Part B 48, 277-302 (2005).

${ }^{29}$ Documentation (2010) Ansys Fluent 12.0/12.1 (Ansys Inc., Canonsburg, Pennsylvania USA).

${ }^{30} \mathrm{M}$. Westerburg and F. H. Busse, "Finite-amplitude convection in the presence of finitely conducting boundaries," J. Fluid Mech. 432, 351-367 (2001).

${ }^{31}$ C. H. Yu, M. Y. Chang, C. C. Huang, and T. F. Lin, "Unsteady vortex roll structures in a mixed convective air flow through a horizontal plane channel : an numerical study," Int. J. Heat Mass Transfer 40, 505-518 (1997).

${ }^{32}$ S. Xin, X. Nicolas, and P. L. Quéré, "Stability analyses of longitudinal rolls of poiseuillerayleigh-bénard flows in air filled channels of finite transversal extension." Num. Heat Transfer Part A 50, 467-490 (2006).

${ }^{33}$ G. E. Willis and J. W. Deardorff, "Roll-diameter dependence in rayleigh convection and its effect upon heat flux," J. Fluid Mech. 54, 351-367 (1972).

${ }^{34}$ F. H. Busse and R. M. Clever, "Instabilities of convection rolls in a fluid of moderate Prandtl number," J. Fluid Mech. 91, 319-335 (1979). 